\title{
RETRACTED ARTICLE: Hybrid Cascode Miller Compensation with Bandwidth Extension for 28-nm CMOS Multistage Amplifiers Driving Large Capacitive Loads
}

\section{Chunming Zhang ${ }^{1} \cdot$ Zhanke Yan $^{1} \cdot$ Menghai Wang $^{1}$}

Received: 25 January 2019 / Revised: 4 July 2019 / Accepted: 5 July 2019 / Published online: 23 July 2019

(c) Springer Science+Business Media, LLC, part of Springer Nature 2019

The authors have retracted this article [1]. After constructing the amplifier circuit as proposed in this article, it became apparent that the capacitance range of the capacitor $\mathrm{CL}$ is much smaller than the simulation data in the article. Subsequent analysis revealed that there were errors in the theoretical analysis: the proposed circuit is incomplete and flawed in design and implementation. The data in this article are therefore unreliable. [All authors agree with this retraction.]

\section{References}

1. C. Zhang, Z. Yan, M. Wang, Hybrid Cascode Miller Compensation with Bandwidth Extension for 28-nm CMOS Multistage Amplifiers Driving Large Capacitive Loads. Circuits Syst. Signal Process (2019). https://doi.org/10.1007/s00034-019-01202-1

Publisher's Note Springer Nature remains neutral with regard to jurisdictional claims in published maps and institutional affiliations.

Electronic supplementary material The online version of this article (https://doi.org/10.1007/s00034019-01202-1) contains supplementary material, which is available to authorized users.

Chunming Zhang

zhangchunming07@xupt.edu.cn

Zhanke Yan

yzk_96_ic@163.com

Menghai Wang

menghai_wang@163.com

1 School of Electronics Engineering, Xi' an University of Posts and

Telecommunications, Xi' an 710121, China 